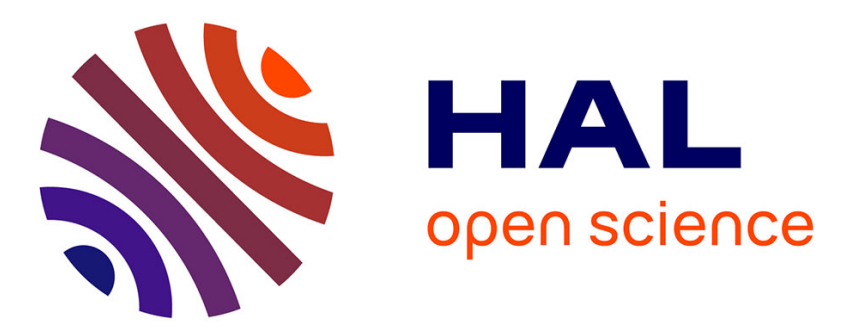

\title{
Optical amplification and its saturation in semiconductor quantum wells
}

\author{
J. Butty, G. Bongiovanni, J. Staehli
}

\section{To cite this version:}

J. Butty, G. Bongiovanni, J. Staehli. Optical amplification and its saturation in semiconductor quantum wells. Journal de Physique IV Proceedings, 1993, 03 (C5), pp.C5-31-C5-38. 10.1051/jp4:1993506 . jpa-00251592

\section{HAL Id: jpa-00251592 https://hal.science/jpa-00251592}

Submitted on 1 Jan 1993

HAL is a multi-disciplinary open access archive for the deposit and dissemination of scientific research documents, whether they are published or not. The documents may come from teaching and research institutions in France or abroad, or from public or private research centers.
L'archive ouverte pluridisciplinaire HAL, est destinée au dépôt et à la diffusion de documents scientifiques de niveau recherche, publiés ou non, émanant des établissements d'enseignement et de recherche français ou étrangers, des laboratoires publics ou privés. 


\title{
Optical amplification and its saturation in semiconductor quantum wells
}

\author{
J. BUTTY, G. BONGIOVANNI* and J.L. STAEHLI
}

Institut de Physique Appliquée, Ecole Polytechnique Fédérale, PH-Ecublens, 1015 Lausanne, Switzerland

* Dipartimento di Scienze Fisiche, Università degli Studi di Cagliari, via Ospedale 72, 09124 Cagliari, Italy

\begin{abstract}
We present an investigation of the optical gain and its saturation of the electronhole plasma confined in $\mathrm{GaAs} /(\mathrm{Ga}, \mathrm{Al}) \mathrm{As}$ quantum wells. For such investigations the variable stripe length method is a simple but quite powerful tool. The spacial longitudinal variation of luminous and electron-hole pair densities in narrow stripes of various lengths has been investigated theoretically and experimentally. For this also the spectra of spontaneous luminescence (emitted in a direction perpendicular to the stripe) have been recorded at different places on the stripe. The experimental results are compared with the predictions of a theoretical model of the optical quantum well amplifier. We find that saturation is caused by carrier depopulation through stimulated electron-hole recombination, and by the loss of light travelling in the direction of the stripe due to scattering and/or reabsorption. However, we do not find evidence of intra band saturation, ie. of a non thermal electron or hole distribution.
\end{abstract}

\section{INTRODUCTION}

Nowadays, there is a considerable interest in the optical properties of semiconductor quantum well (QW) structures, for their potential use in optoelectronic systems, and especially for making compact laser sources. A thorough understanding of the electronic and optical properties of strongly excited QW [1] is therefore crucial for the development of such lasers. In this paper we present an investigation of the amplified spontaneous spectra using the variable stripe length method introduced by Shaklee and coworkers [2]. With this method investigations can be made in the weak as well as in the strong excitation regimes, ie. optical gain and its saturation $[2,3]$ can be studied. The excited stripe being a mirrorless single pass laser, the results obtained in this geometry can directly be applied to practical lasers.

Optical gain spectra of strongly excited semiconductor samples can be obtained in different ways:

(i) The measurement of transmission spectra using a pump and probe method [1] is usually done in a direction perpendicular to the plane of the interesting epitaxial layers. It has the disadvantage of requiring a high number of QW (typically 100) which makes uniform excitation difficult; further only quite high gain coefficients can be determined.

(ii) The observation of the depth of modulation caused by Fabry-Perot interference of the luminescence light emitted in a sample having two parallel and partially reflecting surfaces allows to determine the gain spectra below lasing threshold, a method developed by Hakki and Paoli [4]. 
(iii) Henry and co-workers [5] deduced the gain spectra of a lasing structure from the spectra of spontaneous luminescence through the vanRoosbroeck-Shockley (VRS) relation [6], assuming that the carriers are in quasi thermal equilibrium [7]. The chemical potential can be determined from the shape of the luminescence spectra, and/or from the energy of the applied bias.

(iv) In the variable stripe length method [2], used in this work and described with more details later, the optical gain is derived from the increase of the amplified luminescence (AL) intensity with the length of the excited stripe [8]. It allows to measure small to moderately high gain coefficients, and to investigate also the saturation of optical amplification.

(v) Finally, as has been demonstrated recently [9], differential photoluminescence excitation spectroscopy, which is a combination of a pump and probe experiment with excitation spectroscopy, can be used to determine gain and absorption of structures containing only a few QW.

\section{THE ONE-DIMENSIONAL OPTICAL AMPLIFIER}

We consider a wave guiding structure as outlined in figure 1. The sample is illuminated from the top by laser light of intensity $I_{e x c}$ on a surface having the shape of a stripe of width $b$ and length $L$. This laser light creates eh pairs inside the QW situated in the central or active layer. The spontaneous recombination radiation emitted in the direction of the stripe can induce stimulated radiative eh recombination and thus there are two amplified guided waves symmetrically propagating in the $+x$ and $-x$ directions.

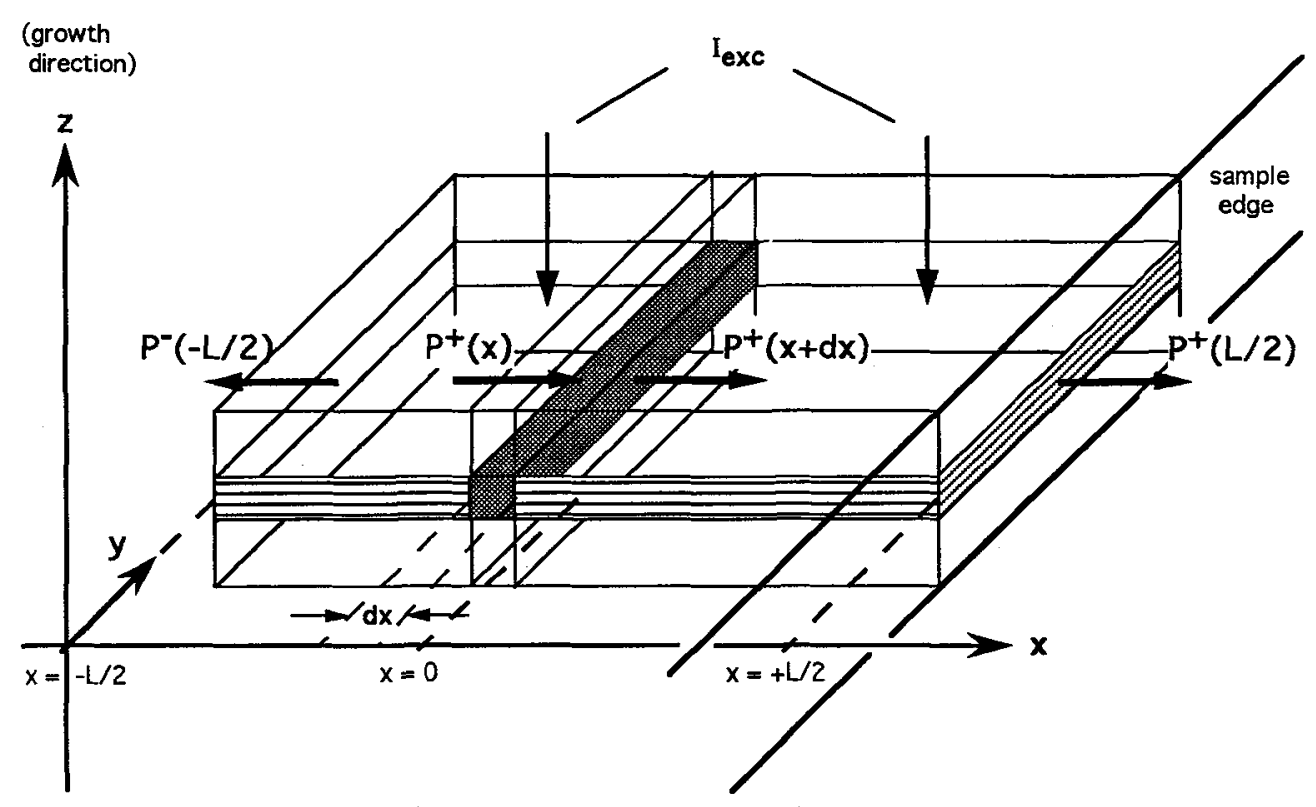

Figure 1: Sketch of the sample excited by laser beam focussed to a rectangular stripe. The hatched area represents the active layer of the wave guiding structure, it consists of a multi quantum well structure. The wells are of $\mathrm{GaAs}$, the barriers and the cladding layers are of $\mathrm{Ga}_{1-\alpha} \mathrm{Al}_{\alpha} \mathrm{As}$ alloy having an $\mathrm{Al}$ concentration $\alpha$ of $50 \%$. The propagation of the amplified luminescence wave $P^{+}(x)$ through an element of length $d x$ is indicated. 
For the power (per unit energy) $P^{+}(h \nu, x)$ of the wave propagating in the $+x$ direction we write

$$
d P^{+}(h \nu)=P^{+}(h \nu, x)[g(h \nu, x)-K] d x+\Omega p_{s p}(h \nu, x) d x
$$

where $h \nu$ is the energy of the travelling photons, and $\Omega p_{s p}(h \nu, x)$ is the power per unit length (and per unit energy) of the spontaneous light emitted into the modes contributing to the amplified wave. Equation (1) defines the optical gain coefficient $g(h \nu, x)$ and the loss coefficient $K$ (losses are eg caused by sample imperfections), $g(h \nu)$ will be relatively small because the major part of $P(h \nu)$ travels outside the excited QW. The light intensity $I(h \nu)$ (per unit energy) which induces the eh transitions in the wells is approximately given by

$$
I(h \nu) \simeq \frac{P(h \nu) C}{b h}
$$

where $C$ is the confinement factor of the propagating mode and $h$ is the height of the central layer. In our case $h$ is small and in the vertical direction there can be only one TE and and TM mode [10].

We assume steady state conditions, ie. for the eh pair density $n(x)$ (per unit surface) at the place $x$ we have in each well

$$
\frac{\partial n(x)}{\partial t}=0=-\frac{n(x)}{\tau_{s p}}-\frac{1}{N b} \int \frac{g(h \nu, x) P(h \nu, x)}{h \nu} d(h \nu)+G .
$$

The first two terms on the right of equation (3) describe the loss of eh pairs by spontaneous and stimulated recombination, $\tau_{s p}(n)$ is the total spontaneous recombination lifetime, $N$ is the number of wells in the central layer, $P(h \nu) \equiv P^{+}(h \nu)+P^{-}(h \nu)$, and $G$ is the rate of eh pairs (per unit surface) generated by the pump.

The density $n(x)$ and the luminescence spectra $p_{s p}(h \nu, x)$ and $P(h \nu, x)$ depend on the position $x$ on the stripe, their profiles will be symmetric with respect to the centre of the stripe $(x=0)$ as long as no light is reflected back into the stripe at the sample edge. As long as the carriers are in a quasi thermal equilibrium, from their density $n(x)$ the corresponding spontaneous luminescence spectra can approximately be obtained through $[11,12]$

$$
p_{s p}(h \nu, x) \approx \sum_{h} \frac{h \nu N b}{\tau_{s p}^{(h)}} D^{(h)}(h \nu, x) f_{e}\left(E_{e}, \mu_{e}(x)\right) f_{h}\left(E_{h}, \mu_{h}(x)\right),
$$

and the gain spectra through the van Roosbroeck-Shockley (vRS) relation [6],

$$
g(h \nu, x)=\frac{\pi^{2} c^{2} \hbar^{3} C}{n_{w g}^{2}(h \nu)^{2}} \frac{p_{s p}(h \nu, x)}{h \nu b h}\left(\exp \frac{h \nu-\mu(x)}{k_{B} T}-1\right)
$$

In (4) and (5), $1 / \tau_{s p}^{(h)}$ ( $h$ stands for heavy holes or for light holes) is proportional to the square of the transition matrix element (assumed to be constant), $D^{(h)}(h \nu, x)$ is the joint density of states, $f_{a}\left(E_{a}, \mu_{a}(x)\right)(a=e, h)$ are the Fermi distribution functions for the electrons and the holes, $E_{a} \equiv$ $\left(h \nu-E_{n n}\right) m_{e h} / m_{a}$ are the kinetic energies of the electrons and holes involved in a transition of energy $h \nu, E_{n n}$ is the gap between the $n$th electron and hole subbands, $m_{e h}$ is the reduced mass of an eh pair, $\mu(x) \equiv \mu_{e}(x)+\mu_{h}(x)$ is the total chemical potential of the eh plasma, $n_{w g}$ is the refractive index of the wave guiding structure, and $T$ is the effective carrier temperature.

Collision broadening was also taken into account, as spectral function for the eh pair states we used

$$
A_{e h}\left(h \nu, E_{e h}(k)\right)=\frac{1}{2 \Gamma_{e h}}\left(1-\tanh ^{2} \frac{E_{e h}(k)-h \nu}{\Gamma_{e h}}\right),
$$


with $E_{e h}(k)=\hbar^{2} k^{2} /\left(2 m_{e h}\right)$ and a constant width $\Gamma_{e h} \approx \Gamma_{e}+\Gamma_{h}$. With this, one obtains for the joint density of states

$$
D^{(h)}(h \nu)=\frac{1}{2 \pi^{2}} \int d^{2} k A_{e h}(h \nu, E(k)) \sum_{n} \Theta\left(h \nu-E_{n n}\right)=\sum_{n} \frac{m_{e h}}{2 \pi \hbar^{2}}\left(1+\tanh \frac{h \nu-E_{n n}}{\Gamma_{e h}}\right) .
$$

Taking the spectral function given by equation (6) instead of the usual Lorentzian has the advantage that the exponential tails $[12,13,14]$ we observe in the optical spectra appear also in $D^{(h)}(h \nu)$ given by (7). Finally, also the reductions of the band gaps have been accounted for, they have been taken to be proportional to $n^{1 / 3}$ [15], the proportionality constants have been chosen to reproduce as well as possible the theoretical results [16].

In the limit of weak excitation and/or short stripe lengths $L$, the rate of stimulated transitions (second term on the right of equation (3)) remains much smaller than the rate of spontaneous transitions $n(x) / \tau_{s p}$, and therefore $g$ and $p_{s p}$ do not depend on $x$. For this case equation (1) can easily be integrated [2]:

$$
P^{+}(h \nu, L)=\frac{\Omega p_{s p}(h \nu)}{g(h \nu)-K}\{\exp [(g(h \nu)-K) L]-1\} .
$$

This result has widely been used to determine the gain spectra $g(h \nu)$, by fitting a series of AL spectra $P(h \nu, L)$ measured for different stripe lengths $L$ to equation (8) [2]. $p_{s p}(h \nu)$ can either be treated as an independent fitting parameter, or can be replaced using the vRS relation (5), or can, in the best case, be measured (up to a constant) [17]. The power $P^{+}(h \nu, L)$ of the AL increases about exponentially with $L$, but only for short stripes. Once $g L \geq 5$ [18], one observes an increase of $P^{+}(h \nu, L)$ which is slower than that given by $(8)$, and at certain wavelengths after an initial increase it even decreases again. This saturation of the optical amplification is, to an important extent, due to carrier depopulation by stimulated eh recombination. More general, numerical solutions of equations (1) and (3) including simple models of saturation can be found in literature [19, 20].

With the simplifications mentioned above, we solved (1) and (3) numerically and selfconsistently for different generation rates $G$ and stripe lengths $L$, the temperature $T$ and the spectral width $\Gamma_{e h}$ have been taken from the high and low energy tails, respectively, of the experimental spectra. The resulting profiles of luminous power and carrier density and are shown in figure 2 .

The total integrated AL power, $P^{t o t}(x) \equiv \int d(h \nu)\left(P^{+}(h \nu, x)+P^{-}(h \nu, x)\right)$, and the total emitted spontaneous luminescence power per unit length, $p_{s p}^{\text {tot }}(x) \equiv \int d(h \nu) p_{s p}(h \nu, x)$, have complementary profiles. While $p_{s p}^{t o t}(x)$ has a maximum at the centre and decreases going toward the ends of the stripe, $P^{\text {tot }}(x)$ has a minimum for $x=0$ and increases with $x$. The two amplified waves, $P^{+t o t}(x)$ and $P^{-t o t}(x)$, are strongest at the ends of the stripe after having travelled a long way through the excited area. The high intensity of the AL in turn induces a high eh recombination rate which causes carrier depopulation (see figure 2c) and thus a decrease of the total spontaneous luminescence at the ends of the stripe; the calculated eh pair density drops from about $1.0 \cdot 10^{12} \mathrm{~cm}^{-2}$ at the centre of the stripe to $0.7 \cdot 10^{12} \mathrm{~cm}^{-2}$ at $x \simeq 1.5 \mathrm{~mm}$. The $x$ dependence of $p_{s p}^{\text {tot }}, P^{t o t}$ and $n$ is quite pronounced close to their extrema at $x=0$, while for longer stripes and $x$ close to $L / 2$ luminescence and carrier density remain almost constant. This leveling off is caused by the losses of AL, for very strong $\mathrm{AL}, P^{t o t}(x) K$ becomes comparable to the pump power; in fact for sufficiently big $L, P^{t o t}(x \simeq L / 2)$ becomes independent on $L$, as can be seen in figure 3 . If these losses are not accounted for, there is no leveling off in the profiles (in particular for the stimulated emission), and a slow continuous change is obtained also for large $x[19]$.

\section{COMPARISON BETWEEN THEORY AND EXPERIMENT}

The sample investigated in our experiments contains five $10 \mathrm{~nm}$ wide GaAs QW separated by 20 $\mathrm{nm}$ thick $\mathrm{Al}_{0.50} \mathrm{Ga}_{0.50}$ As barrier layers. The top and bottom $\mathrm{Al}_{0.50} \mathrm{Ga}_{0.50}$ As layers are $1 \mu \mathrm{m}$ thick, 


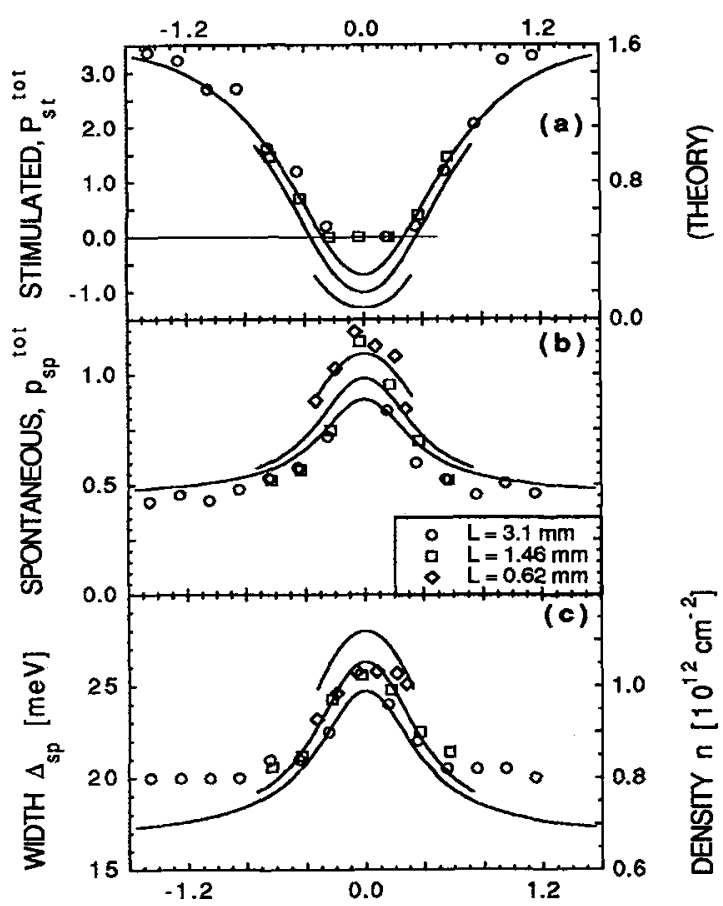

POSITION $x$ ON THE STRIPE [mm].
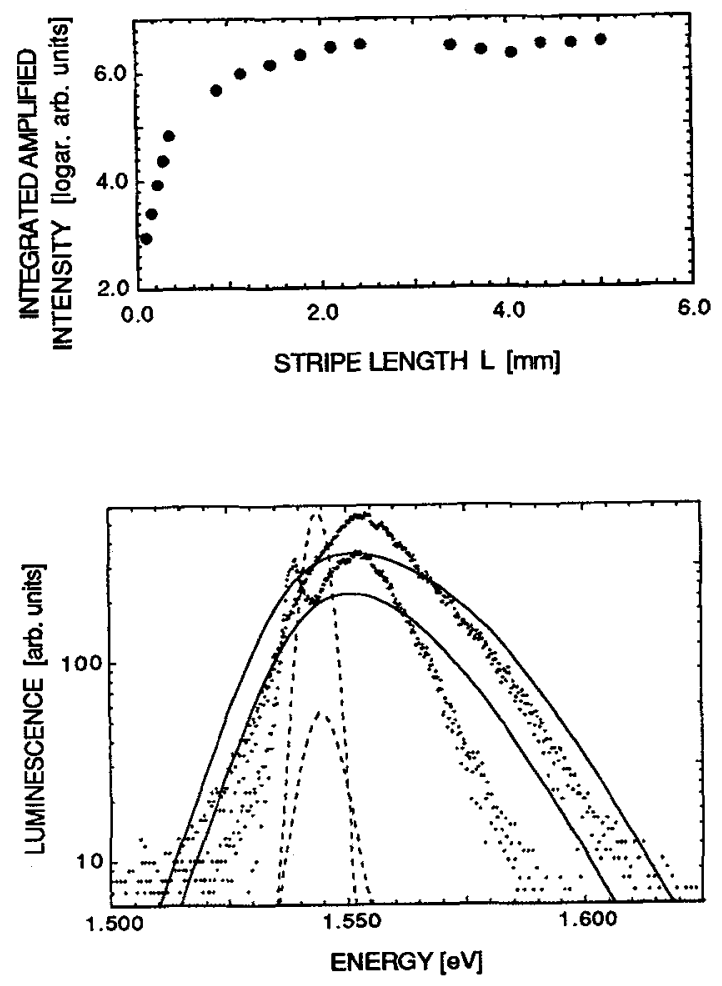

Figure 2. Profiles (a) of the total integrated $\mathrm{AL}$ power $P_{s t}^{\text {tot }},(\mathrm{b})$ of the integrated spontaneous luminescence power $p_{s p}^{t o t}$, and (c) of the measured spectral width $\Delta_{s p}$ of the spontaneous emission, for three different stripe lengths $L$ (the curves extend from $-L / 2$ to $+L / 2$ ). Dots (left scales) are experimental data, they have been measured at a bath temperature of $2 \mathrm{~K}$ and an excitation intensity of $30 \mathrm{kWcm}^{-2}$. The curves (right scales) have been calculated using the model described in the text. The luminescence intensities are given in arbitrary units. In part (c) the widths $\Delta_{s p}$ are compared to the eh pair density $n$, since approximately $n \propto \Delta_{s p}$ [21]. The calculations have been done assuming the following numerical values: losses $K=32 \mathrm{~cm}^{-1}$, generation rate $G=6.5 \cdot 10^{20} \mathrm{~cm}^{-2} \mathrm{~s}^{-1}$ per well, spontaneous recombination lifetimes $\tau^{(h h)}=0.56 \mathrm{~ns}$ and $\tau^{(l h)}=$ $1.7 \mathrm{~ns}$, collision broadening parameter $\Gamma_{e h}=6$ $\mathrm{meV}$, carrier temperature $T=115 \mathrm{~K}$.

Figure 3. Logarithmic plot of the measured integrated amplified luminescence intensity $P^{+t o t}$ versus stripe length $L$, for an excitation intensity of $30 \mathrm{kWcm}^{-2}$. For $L$ bigger than about $2 \mathrm{~mm}, P^{+ \text {tot }}$ is independent of $L$. From the first five points, an integrated gain coefficient of $73 \mathrm{~cm}^{-2}$ can be determined.

Figure 4. Measured spontaneous luminescence spectra (dots), and calculated spontaneous (solid lines) and amplified (dashed lines) luminescence spectra, for a stripe length $L$ of $3.1 \mathrm{~mm}$. The wide spectra are for $x=0$ (centre of the stripe), and the narrow ones for $x \simeq 1.5 \mathrm{~mm}$. The narrow peak that can be observed at about $1.54 \mathrm{eV}$ is due to the intense amplified luminescence travelling in the layer plane which is scattered by sample imperfections. The parameters used for the computations are those given in the caption of figure 2. 
thus the whole structure acts as a wave guide. The sample was excited by the frequency doubled emission of a Nd:YAG (yttrium aluminium garnet) laser ( $\hbar \omega=2.33 \mathrm{eV}$, pulse duration $50 \mathrm{~ns}$ ), with a maximum intensity of $100 \mathrm{kWcm}^{-2}$. The excited stripe was 0.1 to several mm long and $50 \mu \mathrm{m}$ wide, it was slightly tilted with respect to the sample edge, to avoid light reflection back into the excited region. The AL was collected from the edge of the sample while the spontaneous emission was collected from the sample surface in a direction perpendicular to the stripe. In both directions the sample was imaged onto diaphragms to cut away the light coming from weakly excited regions; the diaphragm for the spontaneous emission was a rectangle allowing to observe sample surfaces of 40 by $100 \mu \mathrm{m}^{2}$, and thus the variation of the spectra along the stripe could be detected. The spectra were measured using a diode array equipped with a gateable intensifier. The measurements were performed at bath temperatures of 2,100 and $140 \mathrm{~K}$.

The spectra measured at different lattice temperatures have the same main features, in figure 4 some of the spectra obtained at a bath temperature of $2 \mathrm{~K}$ are shown. In agreement with symmetry, the experimental spectra are practically equal if taken at the same distance $x$ from the centre of the excited stripe. The narrow emission peak on the low energy tail of $p_{s p}(h \nu)$ is due to scattering on crystal defects of the intense amplified light travelling in the well plane; in fact the observed spectral width and energy of this peak correspond closely to those of the emission collected in the direction of the stripe. The experimental gain spectrum of figure 5 was obtained as explained above by fitting the AL intensities measured for different short stripe lengths $L(\leq 0.3 \mathrm{~mm})$ to equation $(8)$.

In figure 2 the dots represent the experimentally determined profiles. There is a reasonable agreement with the theoretical predictions, indicating that our relatively simple model already provides a semiquantitative description of the mirrorless single pass laser. However, some systematic differences between theory and experiment can be observed. The measured profiles of mainly the total spontaneous emission seem to be more pronounced than the calculated ones. In fact, to obtain a profile comparable to what is observed, the carrier density $n(x=0)$ at the centre of the stripe has to be assumed to be somewhat higher than in reality, causing the calculated spontaneous emission to be too wide (see figure 4). Further, the measured gain spectra are always narrower than the observed ones (figure 5), and the experimentally observed high energy tail is always steeper than predicted by theory. Moreover, the peak of AL occurs at lower energy and is narrower than what is calculated.

Finally let us mention that there is no evidence for spectral hole burning in the optical spectra, at least as far as the carrier distribution within a given subband is concerned. This can be understood as follows: The above mentioned leveling off in the luminescence and density profiles happens once the power losses due to light scattering become equal to the power supplied by the pump to generate new eh pairs, ie. when

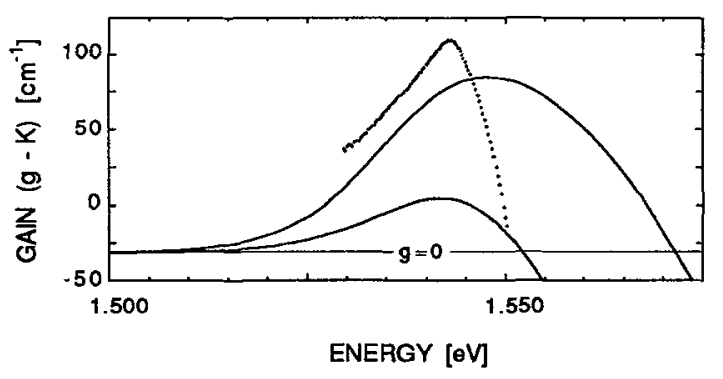

Figure 5. Optical gain spectra: the dots were obtained by measuring the amplified luminescence spectra for different stripe lengths $L \leq 0.3$ $\mathrm{mm}$ and for an excitation intensity of $30 \mathrm{kWcm}^{-2}$. The curves have been calculated for $L=3.1 \mathrm{~mm}$ at $x \simeq 1.5 \mathrm{~mm}$, and for $L=0.3 \mathrm{~mm}$ at $x=0$ (centre of the stripe). The parameters used for the computations are those given in the caption of figure 2 . 


$$
K P^{t o t} \simeq N b G \overline{E_{\text {eh }}},
$$

where $\overline{E_{e h}}$ is the mean energy of an eh pair. $G$ can be estimated knowing that at the centre of a short stripe the stimulated emission is negligibly small, and thus $G \simeq n(x=0) / \tau_{s p}$. Therefore, at complete saturation,

$$
P^{t o t} \simeq \frac{N b \cdot n(x=0) \cdot \overline{E_{e h}}}{K \tau_{s p}} \simeq 0.3 \mathrm{~W}
$$

where in our case $N=5, b=50 \mu \mathrm{m}, K=30 \mathrm{~cm}^{-1}, n \simeq 10^{12} \mathrm{~cm}^{-2}$, and $\tau_{s p}=0.56 \mathrm{~ns}$ [12, 21] has been assumed. On the other hand, the probability that an eh pair undergoes a stimulated recombination can be estimated to (equation (3))

$$
\frac{1}{\tau_{s t}} \approx \frac{g_{s a t} P^{t o t} \Delta_{s p}}{N b h \nu_{s t} n_{s a t} \Delta_{s t}} \approx \frac{g_{s a t}}{K \tau_{s p}} \frac{n(x=0)}{n_{s a t}} \frac{\Delta_{s p}}{\Delta_{s t}} \frac{\overline{E_{e h}}}{h \nu_{s t}} \approx 2.2 \cdot 10^{10} \mathrm{~s}^{-1}
$$

where at saturation (see figure 5 ) the gain $g_{s a t} \approx 45 \mathrm{~cm}^{-1}$, the density $n_{\text {sat }} \approx 0.7 \cdot 10^{12} \mathrm{~cm}^{-2}$, the spectral widths of spontaneous and stimulated emission, $\Delta_{s p} \approx 40 \mathrm{meV}$ and $\Delta_{s t} \approx 8 \mathrm{meV}$, and $h \nu_{s t} \approx \overline{E_{e h}}$. This "stimulated lifetime" $\tau_{s t}$ of about 40 ps is considerably longer than the typical collision lifetime of 0.1 ps [22].

\section{CONCLUSIONS}

We investigated experimentally and theoretically the spacial variation of luminous and eh pair densities in optically excited stripes of various lengths $L$. The profiles of the inversion in the carrier population was directly monitored by measuring the spontaneous luminescence spectra at different places on the excited stripe. Saturation is due to carrier depopulation through stimulated eh pair recombination, and to the losses of light by scattering on sample imperfections. We did not observe non thermal carrier distributions, confirming the prediction of our theoretical model that the light losses prevent the amplified light intensity from reaching very high levels. Therefore the stimulated eh recombination remains always lower than the (thermalising) intrasubband collision rate.

Acknowledgement. Part of this research has been supported by the Fonds National Suisse de la Recherche Scientifique.

\section{References}

[1] S. Schmitt-Rink, DS. Chemla and DAB. Miller: Advances Phys 38, 89 (1989); C. Weber et al., Phys. Rev. B 38, 12748 (1988).

[2] KL. Shaklee, RE. Nahory and RF. Leheny: J. Lumin. 7, 284 (1973).

[3] GP. Agrawal and NK. Dutta, Long-Wavelength Semiconductor Lasers, Chapter 6 (Van Nostrand Reinhold, New York 1986).

[4] BW. Hakki and TL. Paoli: J. Applied Phys. 46 (1975), 1299

[5] CH. Henry, RA. Logan and FR. Merrit: J. Applied Phys. 51 (1980), 3042

[6] HB. Bebb and EW. Williams: Semiconductors and Semimetals, vol. 8, edited by RK. Willardson and AC. Beer (Academic, New York 1972), 181 
[7] P. Blood et al: J. Applied Phys. 70, 1144; RO. Miles et al: IEE Proceedings-J 139 (1992), 33

[8] E. Zielinsky et al: IEEE J. Quantum Electr. QE-23 (1987), 969; Y. Dankner et al: SPIE Vol.1283, Quantum-Well and Superlattice Physics III, edited by GH. Dohler et al (1990), 326; R Cingolani et al: Phys Rev B 42 (1990), 2893

[9] G. Bongiovanni, JL. Staehli and A. Mura: phys. status solidi (b) 173 (1992), 355

[10] HC. Casey and MB. Panish: Heterostructure Lasers Part A (Academic, Orlando 1978)

[11] G. Bongiovanni and JL. Staehli: Solid State Com. 84, 307 (1992)

[12] B. Devaud, F. Clerot, A. Regreny and K. Fujiara: Superlattices Microstruct. 8, 85 (1990).

[13] MP. Kesler and C. Harder: IEEE J. Quantum Electron. QE-37, 1812 (1991).

[14] DB. Tran Thoai and H. Haug: Phys Rev B 47, 3574 (1993)

[15] S. Schmitt-Rink and C. EII: J. Lumin. 30, 585 (1985)

[16] G. Bongiovanni and JL. Staehli: Solid State Com 80, 473 (1991); G. Bongiovanni, JL. Staehli and C. Bosio: Superlattices Microstruct 8, 81 (1990)

[17] J. Butty et al.: Solid State Com. 86, 155 (1993)

[18] JM. Hvam: J Applied Phys 49, 3124 (1978)

[19] E. Goebel, O. Hildebrand and K. Löhnert: IEEE J. Quantum Electron. QE-13, 849 (1977).

[20] P. Schwendimann and Y. Burki: Phys Rev B 46 (1992), 7479

[21] G. Bongiovanni and JL. Staehli: phys. status solidi (b) 173, 365 (1992).

[22] M. Asada, IEEE J. Quantum Electron. QE-25, 2019 (1989). 